\title{
Electricity Storage for Intermittent Renewable Sources
}

\section{Citation}

Rugolo, Jason, and Michael J. Aziz. 2012. Electricity storage for intermittent renewable sources. Energy \& Environmental Science 2012(5): 7151-7160.

\section{Published Version}

doi:10.1039/C2EE02542F

\section{Permanent link}

http://nrs.harvard.edu/urn-3:HUL.InstRepos:11005278

\section{Terms of Use}

This article was downloaded from Harvard University's DASH repository, and is made available under the terms and conditions applicable to Open Access Policy Articles, as set forth at http:// nrs.harvard.edu/urn-3:HUL.InstRepos:dash.current.terms-of-use\#OAP

\section{Share Your Story}

The Harvard community has made this article openly available.

Please share how this access benefits you. Submit a story.

Accessibility 


\section{Electricity Storage For Intermittent Renewable Sources}

Jason Rugolo, Michael J. Aziz ${ }^{\dagger}$

Harvard School of Engineering and Applied Sciences, 29 Oxford St., Cambridge, MA, USA 02138

keywords: electricity storage; energy; intermittent renewable; wind; solar; photovoltaic; maximum storage power; maximum storage energy; efficiency.

${ }^{\dagger}$ Corresponding Author. maziz@harvard.edu. phone: +1 (617) 495-9884. fax: +1 (617) 495-9837.

\section{Abstract}

With sufficient electricity storage capacity, any power production profile may be mapped onto any desired supply profile. We present a framework to determine the required storage power as a function of time for any power production profile, supply profile, and targeted system efficiency, given the loss characteristics of the storage system. We apply the framework to the electrochemical storage of intermittent renewable power, employing a simplifying linear response approximation that permits the entire efficiency behavior of the system to be described by a single scalar figure of merit-the discharge power capacity. We consider three exemplary grid supply scenarios: constant, grid-minus-baseload, and square wave; and two different production scenarios: wind with a capacity factor $32.5 \%$, and solar photovoltaic (PV) with a capacity factor of $14 \%$. For each of these six combinations of scenarios, the storage energy and discharge power capacity requirements are found for a range of system efficiencies. Significantly diminishing efficiency returns are found on increasing the discharge power capacity. Solid-electrode batteries are shown to have two orders of magnitude too little energy to power ratio 
to be well suited to the storage of intermittent renewable power. For both wind and $\mathrm{PV}$, for all supply scenarios studied, installing $1 \mathrm{MW}$ of peak storage per MW of peak production yields system efficiencies of 70\%-90\%.

\section{Introduction}

The intermittency of renewable power sources such as wind and photovoltaic (PV) presents a major obstacle to their extensive penetration into the grid [1]. The developed world has become accustomed to reliable, on-demand electricity; most of its population simply would not accept access to electricity only when the wind is blowing or the sun is shining. The only way to turn naturally fluctuating wind or PV electricity into a dispatchable electricity source (see Fig. 1) is to have some other dispatchable form of electrical energy to take up the slack (a so-called "balancing capacity").

The intermittency of the renewable sources added to the grid up to the present time have been balanced by the dispatchable forms of electricity (mainly natural gas peaking plants) that currently sit ready to produce when the price incentivizes them to do so [7]. To integrate intermittent sources beyond some level of penetration, the grid will require new balancing capacity. The amount of backup dispatchable power required to balance an intermittent source is unclear, especially in light of the complications associated with transmission availability. Preliminary estimates of the cost of integration vary from $0.006 \mathrm{USD} / \mathrm{kWhr}$ for $20 \%$ penetration to $0.0018 \mathrm{USD} / \mathrm{kWhr}$ for $4 \%$ penetration [7]. Even the low-cost estimates 

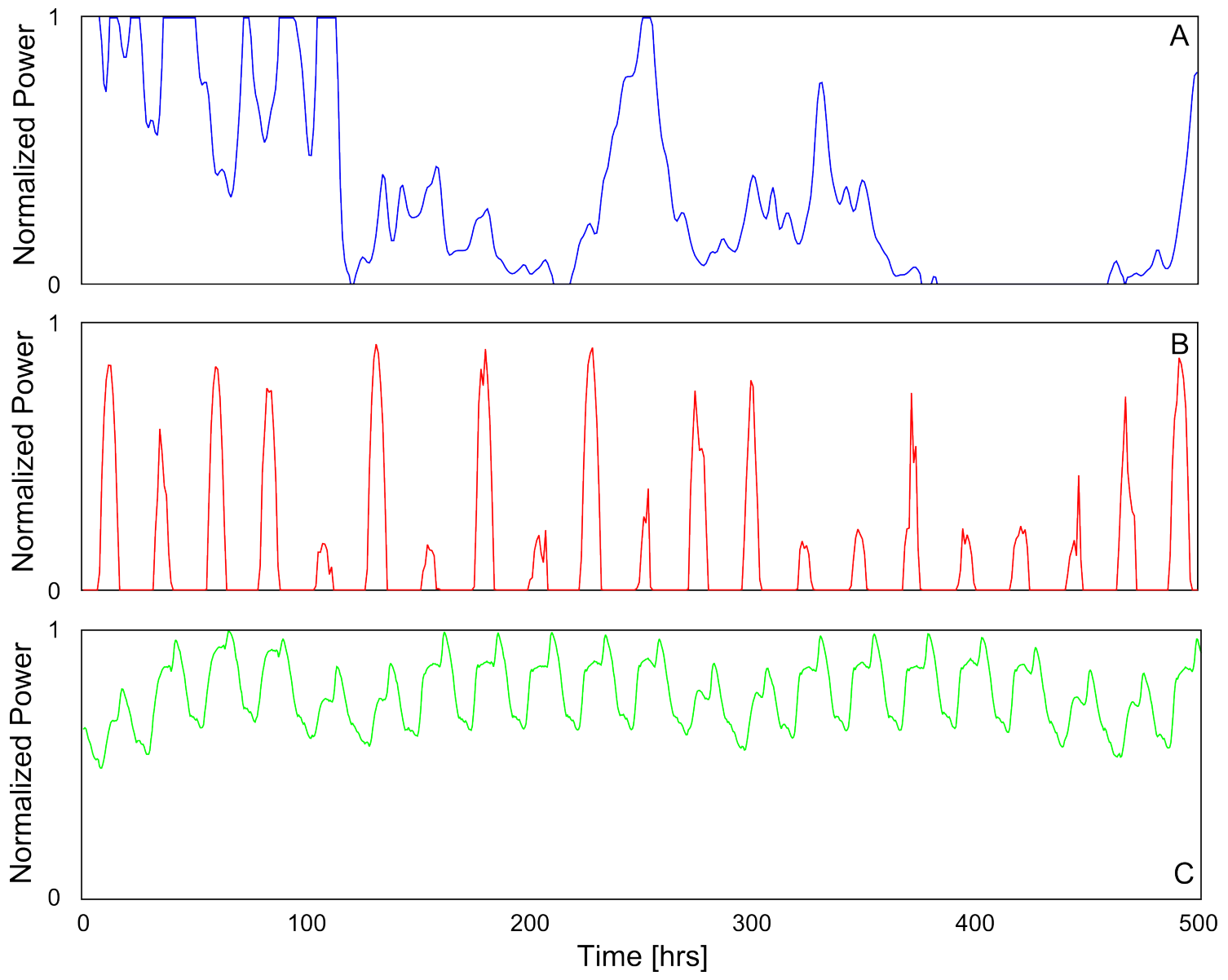

Figure 1: (A) The normalized shape of wind power calculated from real wind speed data in Wilhelminadorp, Netherlands, by applying the power curve for a GE 1.5MW turbine [2, 3, 4]. (B) The normalized shape of solar PV power output in January in Boston, MA, USA [5]. (C) The normalized shape of electricity demand in the UK from the National Grid website [6]. N.B. The data shown are a 500 hour subset of the larger range used for normalization. 
make integration a difficult question. ${ }^{1}$

The potential solutions to the integration problem have their own significant drawbacks. Adding natural gas peaking capacity results in a paradox. The primary motivation for adding renewables in the first place is to lower the carbon intensity of electricity production. By requiring additional natural gas as a backup to smoothen renewable production, a limit is set on the fraction of electricity that can come from the carbon-free sources. ${ }^{2}$

It has been suggested that spatially uncorrelated wind electricity production may have a balancing effect of its own. Electricity can be overproduced where the wind is blowing and be transmitted some distance of order the uncorrelated wind distance (more than several hundred kilometers [8]) to meet demand in becalmed locations. To solve the majority of the problem with this approach, though, appears quite difficult. Nationwide transmission capacity would have to be overbuilt, over very long distances, driving the transmission capacity factor down substantially. This is an expensive proposition which, when added to the already-high price tag of intermittent renewable production, might drive the price of intermittent renewables unacceptably high. Additionally, a grid re-engineering of sufficient magnitude may be indefinitely delayed because it would require massive capital investments and coordination among federal, state, and local governments, private corporations, and private land owners. Certainly something must

\footnotetext{
${ }^{1}$ An upper limit on the integration cost is the cost of an equal amount of dispatchable power for times when the wind is dead or the sun is down.

${ }^{2}$ This limit is complicated, but in the case of $100 \%$ intermittent renewables with natural gas backup and no curtailment, natural gas electricity would constitute one minus the average intermittent renewable capacity factor (something certainly more than half).
} 
be done about the intermittency if President Obama's stated goal of $80 \%$ renewable penetration by 2035 is to be met.

\section{Electricity Storage as a Potential Solution}

Electricity storage (ES) represents a large class of technologies with the potential to address the intermittency problem without a significant marginal carbon footprint. Many types of ES exist, all which have the common characteristic that they convert electricity into stored energy in some medium through a conversion device, and then, either through the same device or another, convert that stored energy back into electricity, while losing some in the round trip due to dissipative processes. Among them are pumped hydro, compressed air, flow batteries, solid-electrode batteries, liquid batteries, regenerative fuel cells, and hybrid electrochemical cells.

Each of these applications provides the opportunity for a rich discussion, with many unique characteristics affecting the ultimate conclusion of whether or not the application's value can overcome the cost of storage and provide an acceptable profit. The economic question is very complicated, as it requires the intersection of a detailed technical understanding of the storage device with a profound understanding of the markets, most of which have yet to be demonstrated on any significant scale. ${ }^{3}$ An in-depth economic analysis requires answers to questions

\footnotetext{
${ }^{3}$ The lack of wide adoption of electricity storage is most commonly attributed to the cost of storage being too high, but this may change in the near future because of the intense research and development currently focused on making ES better and cheaper.
} 
such as how much storage to match to a given intermittent source, what efficiency can be expected, and what are the costs. We briefly discuss each of these in turn.

The required amount of a storage technology depends on the producer's choice for the time-structure of the power to be supplied. For example, the supplied power can be designed to provide constant output, follow the load, or provide constant output only when demand and prices are high. There is no unique answer to this supply shape question because it can depend largely on the local regulatory framework or incentive structures surrounding a specific project.

The efficiency that can be expected is a surprisingly complicated question. Losses are generally dependent on the instantaneous system power and the unique properties of the specific storage system, including its chosen size as specified by attributes such as peak power capacity and total energy storage capacity. For a chosen temporal supply structure and a specific storage system, one may calculate a histogram of the hours at which the storage system suffers various amounts of loss. The average system efficiency can then be calculated once this loss distribution is determined.

Rough costs of storage depend strongly on the amount of energy and power capacity purchased. Deciding on how much of either to purchase depends on expected revenue, which is intimately related to the chosen time-structure of the power to be supplied, and how much energy is lost in the storage process. The energy and power capacity decision is thus characterized by having several codependent parts.

A full economic model is beyond the scope of this work and may not yet be 
possible. However, we expect a generic, economically focused consideration of the technical aspect of ES to be useful for those who may make detailed economic models in the future. Here, we develop a broadly applicable theoretical framework that facilitates the detailed discussion of ES prospects.

\section{Electricity Storage Power Functions}

\subsection{Production, Storage, and Supply Functions}

The function central to our analysis is the power of the ES system as a function of time, which we denote $S T(t)$ (for storage power). $S T(t)$ is positive when the storage system is delivering energy to the consumer (discharging) and it is negative during overproduction when energy is being added to the storage system (charging).

The expected power production profile $P R(t)$ is the power produced by the wind turbine or solar panel (Figure 1A,B); this power is either delivered to the storage system or to the grid, or is lost by dissipation. $P R(t)$ is set by the energy resource and the production system. Only the shape of this profile matters, so for simplicity this function is normalized to unity at its peak value, e.g. the nameplate power production capacity of the wind turbine or PV array.

The supply profile $S U(t)$ is the time-structure of the power that the producer delivers to the grid; it, too, is normalized to unity at its peak value. This represents a specific scenario for how the producer intends to deliver power to the consumer. The supply profile, combined with a model of the time-dependence of 
the electricity price, determines the revenue earned by the production and ES system. Among the possible supply profiles are constant output, load-following (Figure $1 \mathrm{C})$, square wave of various duration and phase, variable profit-maximizing schemes, and many others including combinations of these.

In the absence of dissipative losses, one would scale the supply output such that the area under $S U(t)$ equals the area under $P R(t)$. But losses cause the total energy supplied to be less than the total energy produced by a factor that is not knowable a priori because it depends on the detailed behavior of $S T(t)$ in conjunction with the detailed behavior of the storage technology. To account for this discrepancy we introduce a scalar, $a$, that scales $S U(t)$ vertically. The actual power supplied is $a \cdot S U(t)$; $a$ is determined by the criterion that the area under $a \cdot S U(t)$ is less than the area under $P R(t)$ by the amount of energy lost through storage.

The ES system must do whatever is necessary in order to ensure that the power produced plus the storage power yields the desired power supplied:

$$
S T(t)=a \cdot S U(t)-P R(t) .
$$

Whereas $S U(t)$ and $P R(t)$ are normalized functions assumed to be pre-determined, we must find a way to solve for $a$ such that the energy balance is correct. Calculating $a$ requires knowledge of the storage system's loss rate as a function of time, $P_{\text {loss }}(t)$, which depends on the instantaneous storage power, $S T(t)$, among other things ${ }^{4}$, depending on the technology. This calculation must be done recursively

\footnotetext{
4"Other things" may include the state of charge, de-activation of catalyst, ageing of other components (membranes, etc.), local temperature fluctuations, and many others. These are typically a function of $S T(t)$ and its history, although not always (as with local temperature).
} 
such that $S T(t)$ and $a$ converge to a mathematically consistent solution.

The total energy lost over a long time period $\Delta t=t_{2}-t_{1}$ is the integral of $P_{\text {loss }}(t)$ over that time period:

$$
E_{\text {loss }}^{\Delta t}=\int_{t_{1}}^{t_{2}} P_{\text {loss }}(t) d t
$$

The required magnitude of $\Delta t$ will be discussed shortly. Requiring the net amount of energy stored over this time period to be negligibly small, the energy dissipated must also be the difference between the energy produced and that supplied:

$$
E_{\text {loss }}^{\Delta t}=\int_{t_{1}}^{t_{2}} P R(t) d t-a \int_{t_{1}}^{t_{2}} S U(t) d t
$$

We now equate the two expressions for $E_{\text {loss }}^{\Delta t}$ (Eqs. 2 and 3) to obtain a second relationship, in addition to Eq. 1, between $S T(t)$ and $a$. These equations comprise a closed set that can be solved numerically for $S T(t)$ and $a$, so long as $P_{\text {loss }}(t)$ is known and depends only on $S T(t)$ and its history.

For the results to be informative, $\Delta t$ must be long enough that the behavior of the system has converged onto some average characteristic behavior. A good proxy for meeting this criterion is to track the behavior of the production capacity factor with increasing $\Delta t$. Once the production capacity factor is observed to approach the average value, it is reasonable to assume that the statistically significant variability in the system has been accounted for in the averaging. For the calculations presented later, $\Delta t$ was chosen to be 744 hours (about 1 month). 


\subsection{The Dissipated Power, $P_{l o s s}(t)$}

The power loss function $P_{\text {loss }}(t)$ describes the power being dissipated within the storage system as heat. This function, which depends on many technology-specific parameters, is one of the key technological considerations affecting energy storage prospects.

Each storage technology has differing loss characteristics. Compressed air storage, for example, uses a compressor to compress air for storage, and delivers the energy by letting the air expand through a turbine. The compressor efficiency depends on the efficiency of the motor that turns the compressor, as well as on the state of charge of the reservoir and on all of the dissipative losses in the compressor system. The motor efficiency and the dissipation within the compressor both depend on the compression rate, which is a function of the power during charging and the number of compressors deployed for the job. Similarly, during discharge, the power loss in the turbine is dependent on the characteristics of the turbine, the number of turbines deployed, and the rate at which air flows through a turbine to create electricity. The engineer is able to pin down the dependence of the power loss function on the relevant engineering and operating parameters in the system.

There are similarly complicated loss functions for each storage technology class. Electrochemical cells are among the simplest. An electrochemical cell has several loss mechanisms (called overpotentials) that contribute to the total overall loss. Namely, there is an activation overpotential for each of two electrodes, an ohmic resistance overpotential, and a mass transport overpotential, all of which 
are functions of the current density. ${ }^{5}$ These combine to make the cell potential deviate from the equilibrium potential during operation. The behavior of a typical electrochemical cell is shown in Fig. 2.

The power dissipated in any electrochemical cell during operation represents a comparison of the energy produced or consumed by the cell to the energy that would be produced or consumed if the operation were thermodynamically reversible (i.e. no dissipation). The power density, $p\left[\frac{\mathrm{mW}}{\mathrm{cm}^{2}}\right]$, is the cell potential $E(i)$ $[\mathrm{V}]$ times the current density $i\left[\frac{\mathrm{mA}}{\mathrm{cm}^{2}}\right]$ :

$$
p=E(i) \cdot i
$$

The overpotentials subtract from the reversible potential, which is also called the equilibrium potential, $E_{e q}$. In the ideal (loss-free) case of zero overpotentials, the power density would be

$$
p_{\text {ideal }}=E_{e q} \cdot i
$$

The loss power density is the difference between $p_{\text {ideal }}$ and $p$ :

$$
p_{\text {loss }}=p_{\text {ideal }}-p
$$

$p_{\text {loss }}$ and $P_{\text {loss }}$ are related to each other by the cell area, as are $S T(t)$ and $p$ :

$$
p=\frac{S T(t)}{A},
$$

${ }^{5}$ One may also add a weakly time-dependent "degradation" overpotential, which catches all of the characteristics of diminishing performance over time. In the present work, we ignore these slow effects. 


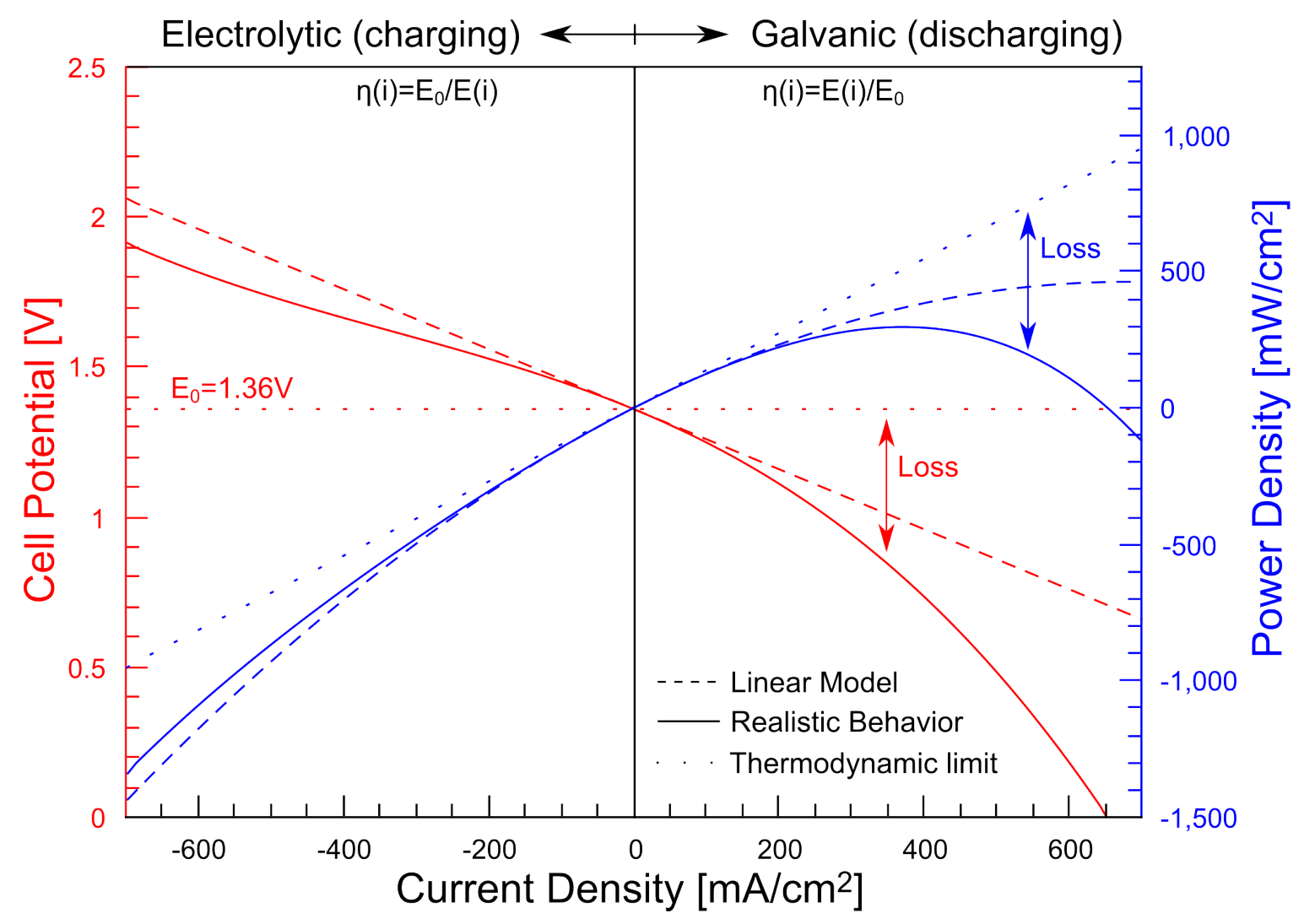

Figure 2: Hydrogen-chlorine regenerative fuel cell behavior exemplifying constant-activity electrochemical cell. In red is the cell potential and in blue is the power density $(p=E(i) \cdot i)$. The zero-current potential in this particular example is $1.36 \mathrm{~V}$ (horizontal dotted line), which is the equilibrium potential under standard conditions for the $\mathrm{H}_{2}(g)$ and $\mathrm{Cl}_{2}(g)$ couple (producing $\mathrm{HCl}(\mathrm{aq})$ ). The solid lines represent realistic fuel cell behavior, the dashed lines represent a linear approximation to the voltage-current relationship, and the dotted lines represent the thermodynamic limit. The total overpotential is indicated by the red arrow as the voltage difference between $E(i)$ and $E_{e q}$. The corresponding power loss is indicated by the blue arrow. 


$$
p_{\text {loss }}=\frac{P_{\text {loss }}(t)}{A}
$$

Because each of the losses is a function of current density, it is important to note that the loss function depends on the total working area of electrochemical cell in operation. 6

The example shown in Figure 2 is that of a hydrogen-chlorine regenerative fuel cell. ${ }^{7}$ This belongs to the larger class of constant-activity electrochemical cells, in which the reactants and products are maintained at constant activity; hence $E_{e q}$ does not depend on the state of charge. ${ }^{8}$ The difference between the equilibrium potential and the cell potential is the magnitude of the voltage loss (the total overpotential), and is made up of the four aforementioned overpotentials. One may see that the loss increases significantly as a function of current density, and in the galvanic direction the loss ultimately consumes $100 \%$ of the cell power.

If $P_{\text {loss }}(S T(t))$ is known, one can solve for $S T(t)$, which fully specifies the prospective ES system. From $S T(t)$, one may derive the system's state of charge $Q(t)$, the efficiency distribution, the amount of energy lost, the maximum stored energy required, the maximum discharge power required, and the general behavior that would be required of the storage system for the chosen delivery scenario. This information is essential for a rational storage selection process.

\footnotetext{
${ }^{6}$ Ultimately, then, the efficiency depends on capital expenditure. For electrochemical cells, the efficiency can get arbitrarily close to $100 \%$ as the installed cell area gets arbitrarily large. The cell area would be a major capital cost parameter in a full economic model.

${ }^{7}$ The hydrogen-oxygen fuel cell is atypical due to the enormous nonlinear oxygen electrode overpotential loss, which makes it unsuitable for efficient ES, and so it is ignored in this work.

${ }^{8}$ Varying-activity cells such as solid-electrode batteries and flow batteries have a $P_{\text {loss }}$ that depends additionally on the state of charge, which must be calculated from the history of $S T(t)$.
} 


\subsection{The Linear Potential Approximation}

The linear potential approximation is a good approximation for most electrochemical cells (with the notable exception of the hydrogen-oxygen fuel cell) and it greatly simplifies the analysis and the ensuing discussion. The actual cell potential illustrated in Figure 2 is more nonlinear than would be expected from a real system-it was chosen that way for illustrative purposes. The approximation is particularly good for high-efficiency operation at low current density, where most cells would be expected to operate for ES applications. Hence we approximate the potential vs. current density as a straight line, permitting an analytical solution for for $P_{\text {loss }}(t)$.

Although in any calculation with high financial stakes one should attempt to model real-world behavior as closely as possible by using a more accurate potential function, even in this case the linear model remains valuable in showing the generic behavior of an ES system. The cell potential as a function of current density is thus described by

$$
E(i)=E_{e q}-\rho i,
$$

where $E_{e q}$ is the equilibrium potential in volts, $i$ is the current density in $\frac{\mathrm{mA}}{\mathrm{cm}^{2}}$, and $\rho$ is the loss parameter in $\mathrm{k} \Omega \cdot \mathrm{cm}^{2}$. The value of $\rho$ for the linear approximation curve in Figure 2 is $0.001 \mathrm{k} \Omega \cdot \mathrm{cm}^{2}$, which is a presently achievable value for this parameter [9].

The power density $p$ can be written solely as a function of current density by substituting Equation 9 into Equation 4. $p$ can also be written solely as a func- 
tion of cell potential by inverting Equation 9 to find $i(E)$, and then substituting $i(E)$ into Equation 4. These manipulations lead to quadratic expressions for $p(i)$ and $p(E)$, respectively. These quadratic equations can be inverted to obtain expressions for both the current density and the potential as functions of the power density. Defining the maximum discharge power density $\Pi$ and the current density $i_{\Pi}$ at maximum power as

$$
\Pi \equiv \frac{E_{e q}^{2}}{4 \rho}
$$

and

$$
i_{\Pi} \equiv \frac{E_{e q}}{2 \rho}
$$

leads the the following compact forms for $i(p)$ and $E(p)$ :

$$
\begin{gathered}
i(p)=i_{\Pi}\left(1-\sqrt{1-\frac{p}{\Pi}}\right) \\
E(p)=E_{e q}\left(\frac{1+\sqrt{1-\frac{p}{\Pi}}}{2}\right)
\end{gathered}
$$

We now solve for $P_{\text {loss }}(S T(t))$ using Equations 5 - 8:

$$
P_{\text {loss }}(t)=2 \Pi A\left(1-\sqrt{1-\frac{S T(t)}{\Pi A}}\right)-S T(t) .
$$

This equation shows that for constant-activity electrochemical cells under the linear potential approximation, the only parameter necessary to characterize the power dissipated versus time for the storage system is the maximum possible discharge power, $\Pi A$. The lost power vs. instantaneous power is plotted for various 
values of this parameter in Figure 3. As $\Pi A$ is increased, the power loss for any given $S T(t)$ scenario decreases. With this expression for $P_{\text {loss }}(S T)$ for a storage technology characterized by $\Pi A$, the set of Equations 1, 2, and 3 forms a closed set from which $S T(t)$ and $a$ can be calculated for any given choice of $P R(t)$ and $S U(t)$.

The product $\Pi A$ in Equation 14 determines the overall efficiency behavior. ${ }^{9}$ Because $\Pi A$ may be chosen freely, we next ask what value of $\Pi A$ is necessary for the overall system efficiency, $\eta_{\text {avg }}$, to reach a targeted value. The average system efficiency is defined as the energy supplied divided by the energy produced over some time period:

$$
\eta_{\text {avg }}=\frac{a \int_{t_{1}}^{t_{2}} S U(t) d t}{\int_{t_{1}}^{t_{2}} P R(t) d t} .
$$

Setting $\eta_{\text {avg }}$ to a target value, e.g. $85 \%$, enables the calculation of the value of $\Pi A$ needed to achieve that average efficiency, for a given $P R(t)$ and choice of $S U(t)$. A plot of $\eta_{\text {avg }}$ as a function of $\Pi A$ provides insight into the cost-benefit tradeoff of installing more storage power.

\section{Production and Supply Scenarios}

Here we examine the storage power and energy requirements for several production and supply scenarios and a range of system efficiencies. The two production scenarios are the intermittent wind and PV temporal profiles shown in Fig. 1 A, B. The first, and simplest, supply scenario is a system supplying constant power

\footnotetext{
${ }^{9} \Pi$ depends on which technology is installed, and $A$ is how much of it is installed.
} 


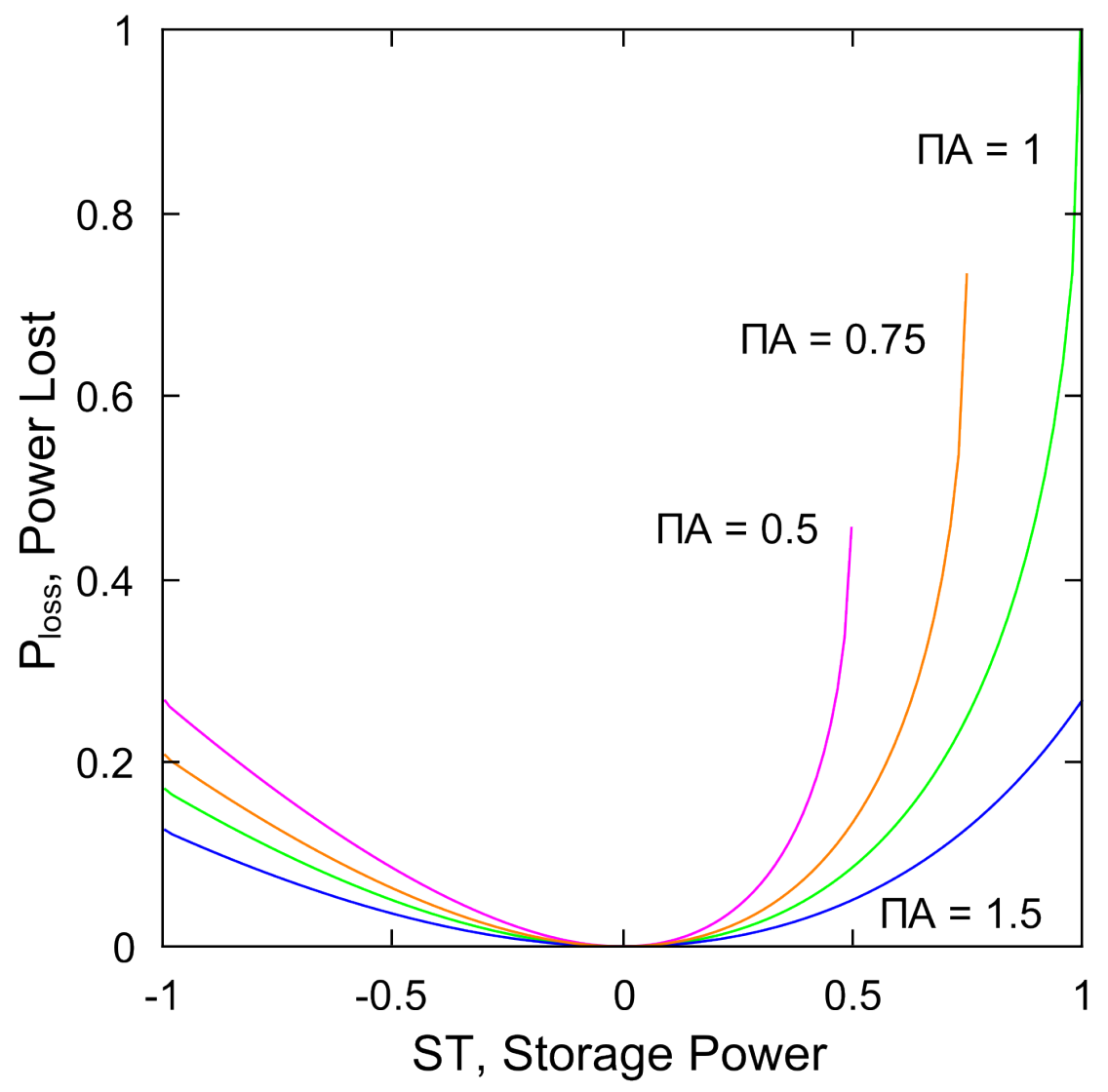

Figure 3: Lost power vs. storage power for different values of the maximum possible discharge power, $\Pi A$. Both power variables are normalized by the source nameplate power. 
from either of these intermittent sources. We subsequently consider a temporal supply structure that follows grid daytime demand, and a square wave that supplies all of its power during a five-hour period of peak demand.

\subsection{Constant Power from Intermittent Wind or PV}

The low capacity factors of intermittent renewables cause a difficult transmission problem. If enough transmission is built to transmit the peak power capacity of an intermittent power source, on average the utilized fraction of the transmission capacity is the production capacity factor, which ranges from 20 to $50 \%$ for wind and from 10 to $20 \%$ for solar PV. In some cases, this has led to the decision to build less transmission than renewable capacity, and to curtail the energy produced when production overloads the available transmission [10]. A constant $S U(t)$ has the potential to best utilize available transmission, because one could design the system such that transmission is always operating at full capacity. What storage power and energy capacities are required to map wind and PV production profiles onto a constant supply profile at a specified system efficiency?

Figure 4 shows the resulting storage functions for the wind-to-constant and solar-to-constant production cases. Because the peak production power has been normalized to unity, all power numbers presented here are as fractions of the peak, or nameplate, power of the real system. Through a storage system with $85 \%$ system efficiency, a $1 \mathrm{MW}$ nameplate wind turbine with a capacity factor of 0.325 must output a constant $0.85 \cdot 0.325=0.276$ MW. This implies $a=0.276$ and our calculations find that the required storage hardware has a peak discharge power 

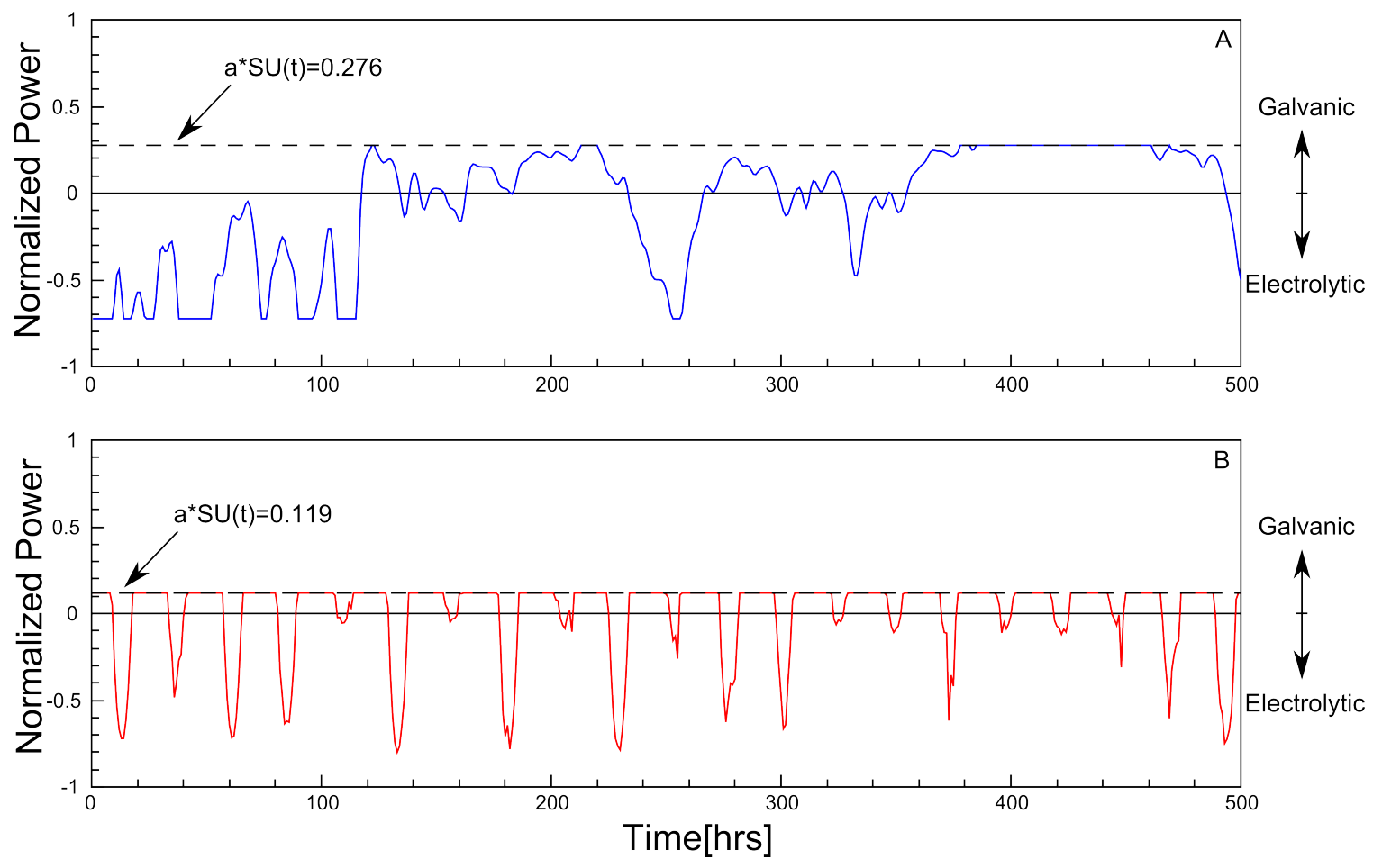

Figure 4: Mapping wind and solar PV sources to constant power output with a specified average system efficiency of $\eta_{\text {avg }}=0.85$. (A) Storage power, $S T(t)$, for the wind production profile $P R(t)$ shown in Figure 1A (capacity factor 0.325 ), that is required for converting wind to constant output $S U(t)=1$. The results are $a=0.276$ and $\Pi A=0.480$ as discussed in the text. (B) $S T(t)$ for the PV PR(t) shown in Figure 1B (capacity factor 0.14), that is required for converting PV to constant $S U(t)=1$. The results are $a=0.119$ and $\Pi A=0.568$ as discussed in the text. Power is normalized by source nameplate power capacity. 
capacity of $\Pi A=0.480$ times the turbine nameplate. Likewise, for PV levelized through a storage system with $85 \%$ system efficiency, a 1 MW peak PV array with a capacity factor of 0.14 must output a constant $0.85 \cdot 0.14=0.119 \mathrm{MW}$. This implies $a=0.119$ and the required storage hardware has a peak discharge power capacity of $\Pi A=0.568$ times the PV peak power. The dashed lines in the figures, showing the constant power supplied in each case $(a \cdot S U(t))$, are consistent with the capacity factors of 0.325 for the wind example and 0.14 for the PV example.

Finally, we determine the energy capacity of the storage needed for these scenarios. First, the state of charge (SOC), $Q(t)$, must be determined as a function of time. This is done by integrating the current from Equation 12 from an arbitrarily chosen time origin:

$$
Q(t)=A \int_{0}^{t} i\left(p\left(t^{\prime}\right)\right) d t^{\prime}=i_{\Pi} A \int_{0}^{t}\left(1-\sqrt{1-\frac{S T\left(t^{\prime}\right)}{\Pi A}}\right) d t^{\prime}
$$

The SOC varies with time in much the same way as the powers do. The total charge capacity required by the storage system is the difference between the absolute maximum SOC and the absolute minimum SOC over all time. Because we need to project the future from data covering a limited time span in the past, we must ensure that this time span is long enough to make reasonably reliable predictions, e.g. that potential extended charge or discharge cycles have been included in the analysis. For example, for wind we must make sure to include a characteristic dead spell, which requires the storage of a large amount of charge. A good proxy for this condition should be tracking the capacity factor as the period of 
integration increases, as discussed above. To calculate the energy capacity ${ }^{10}$, one must multiply the charge capacity by the equilibrium potential, $E_{e q}$.

When we perform this calculation we find that the energy capacity required for the wind-to-constant scenario of Figure 4A is 98 hours, whereas the energy capacity required for the solar-to-constant scenario of Figure 4B is 29 hours-i.e., for $1 \mathrm{MW}$ nameplate wind and PV systems, $98 \mathrm{MWhr}$ and $29 \mathrm{MWhr}$, respectively. Wind outproduces solar by the ratio of their capacity factors-in these scenarios, $0.325 / 0.14=2.32$. This is not as large a ratio as the ratio of the required energy, which is $98 / 29=3.4$. Wind requires more energy capacity in these scenarios, even after accounting for its increased production capacity factor, because there are longer periods of time when the wind does not blow than there are when the sun does not shine, and the storage must be there to take up the slack. These long dead periods create a particularly large energy capacity burden on a storage system.

The required storage power and energy depend on the desired system efficiency. Figure 5A shows the system efficiency, $\eta_{\text {avg }}$, as a function of the maximum discharge power $\Pi A$ for both the wind- and solar-to-constant scenarios in Figure 4. As we increase the system efficiency specification, the necessary maximum power $(\Pi A)$ increases in order to achieve more efficient cell operation. As the system efficiency approaches the limit of $100 \%$, we see diminishing returns on increasing the maximum discharge power. Figure 5B shows the relationship between the required energy capacity and $\eta_{\text {avg }}$ for the same scenarios. Note that the relationship is indirect. The specified system efficiency determines $\Pi A$, as in

\footnotetext{
${ }^{10}$ The energy capacity would more appropriately be called the reversible energy capacity, because it is the energy that would be delivered only in the case of reversible reactions.
} 
Figure 5A. Then, for a given $S T(t)$ scenario, any $\eta_{a v g}-\Pi A$ pair determines a corresponding energy capacity requirement, and this is plotted in Figure 5B.

It is apparent that for the constant supply scenario, storage of wind is much more demanding than storage of solar PV from a storage energy perspective and slightly less demanding from a storage power perspective for most efficiencies. Note that, for flow batteries and regenerative fuel cells, installing extra energy capacity means installing larger tanks and more reactants. This tends to be much cheaper than installing more power capacity.

Both of these storage scenarios permit up to $100 \%$ transmission capacity factor. Thus, for any new installation of intermittent power, one may consider buying less transmission and utilizing it completely, enabled by buying the amount of storage determined above. Also, for existing intermittent power installations that have reached their transmission capacity, storage may be particularly valuable. By adding the amount of storage determined above, the production capacity can be expanded about three times in this wind scenario, and about seven times in this PV scenario, without upgrading the transmission.

\subsection{Time-Dependent Supply Scenarios}

In the same way that the calculations reported above were performed for a constant supply profile $S U(t)=1$, one can evaluate the storage power and energy requirements for any desired supply profile. $S U(t)$ may vary widely within the realm of interest. An $S U(t)$ profile that supplies power only during the peak consumption hours of the day, when the electricity price is high, may be particularly 
profitable. An $S U(t)$ profile that supplies just the peaks of the grid consumptionbut not the baseload power-could represent a synergistic scenario in which intermittent renewable production, combined with a carbon-free baseload production such as nuclear, utilizes electricity storage to provide a carbon-free electricity mix.

We studied three disparate scenarios in order to investigate the variability of the storage system power and energy requirements with varying $S U(t)$. The first is the wind- and solar-to-constant (CONS) scenario described previously. The second is a grid-minus-baseload (GMB) scenario, in which the minimum power consumed is subtracted from the grid profile, as shown in Figure 6B. The third is a square wave (SW), 5 hours in peak width, centered on the daily consumption peak, as shown in Figure 6C. We expect these three cases to provide a sufficiently diverse set of circumstances to provide an idea of how important $S U(t)$ is to the storage requirements. For each of these cases, we calculated both the power and energy requirements for a range of system efficiency values. Diminishing returns at high efficiency are rapidly reached in all cases, as the more detailed results in Figure 5 showed for the CONS scenario. For this reason, we present only the power and energy requirements for system efficiencies $\eta_{\text {avg }}$ ranging from $95 \%$ down to $70 \%$ in increments of $5 \%$. Figure 7 shows the calculation results for the CONS, SW, and GMB supply scenarios.

All the results for the wind production scenario are grouped higher in required storage energy than those for the solar production scenario. The SW supply scenario requires the most power to reach 95\% system efficiency for both wind and solar production profiles. Supplying the CONS scenario requires approximately 

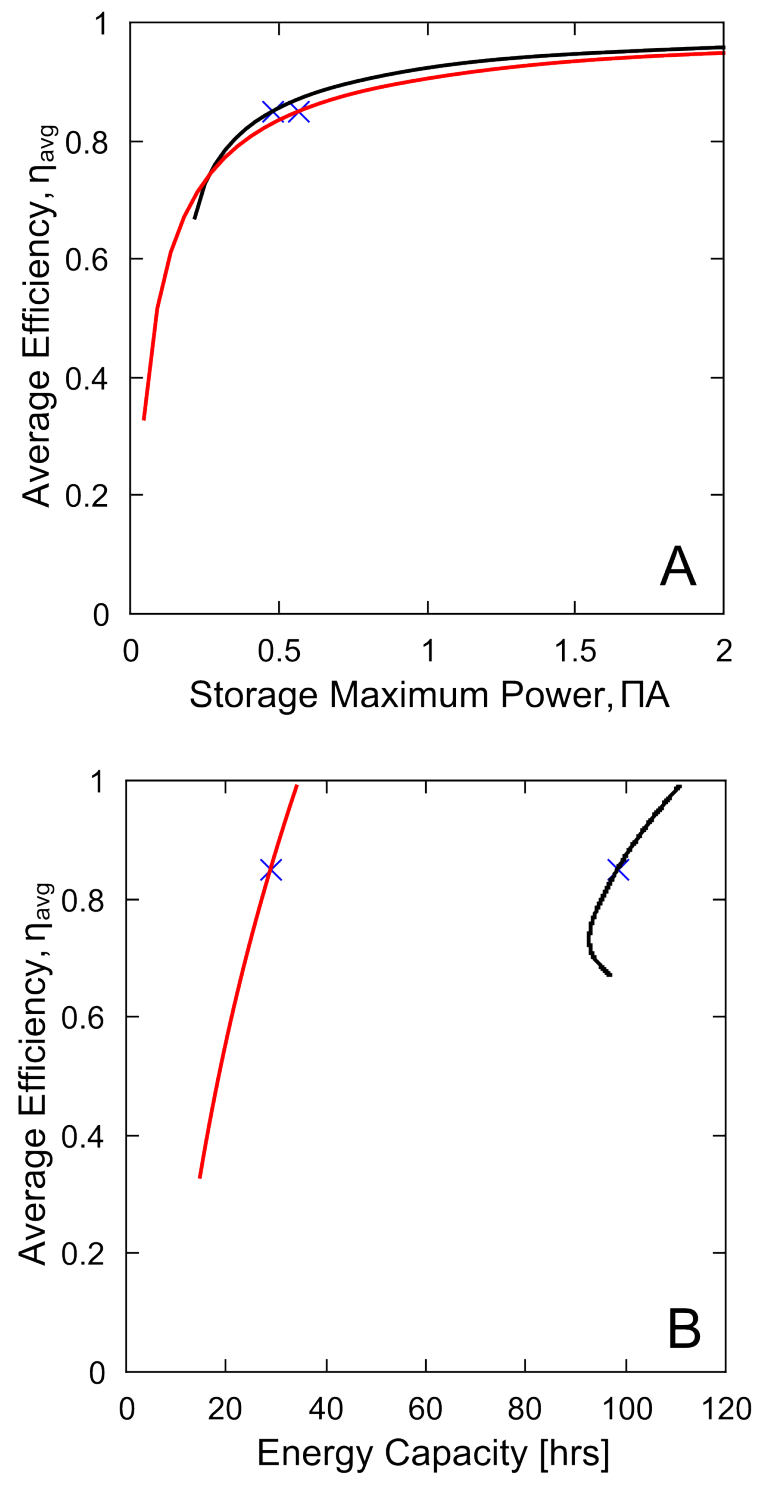

Figure 5: (A) The system efficiency, $\eta_{\text {avg }}$ as a function of the maximum storage discharge power, $\Pi A$, for mapping the wind (black lines) and solar (red lines) scenarios in Figure 1 to a constant output. Power is normalized by source nameplate power capacity. (B) The energy capacities required for the above $\eta_{a v g}-\Pi A$ combinations. The crosses mark $85 \%$ efficiency, corresponding to the $S T(t)$ curves in Figure 4. 

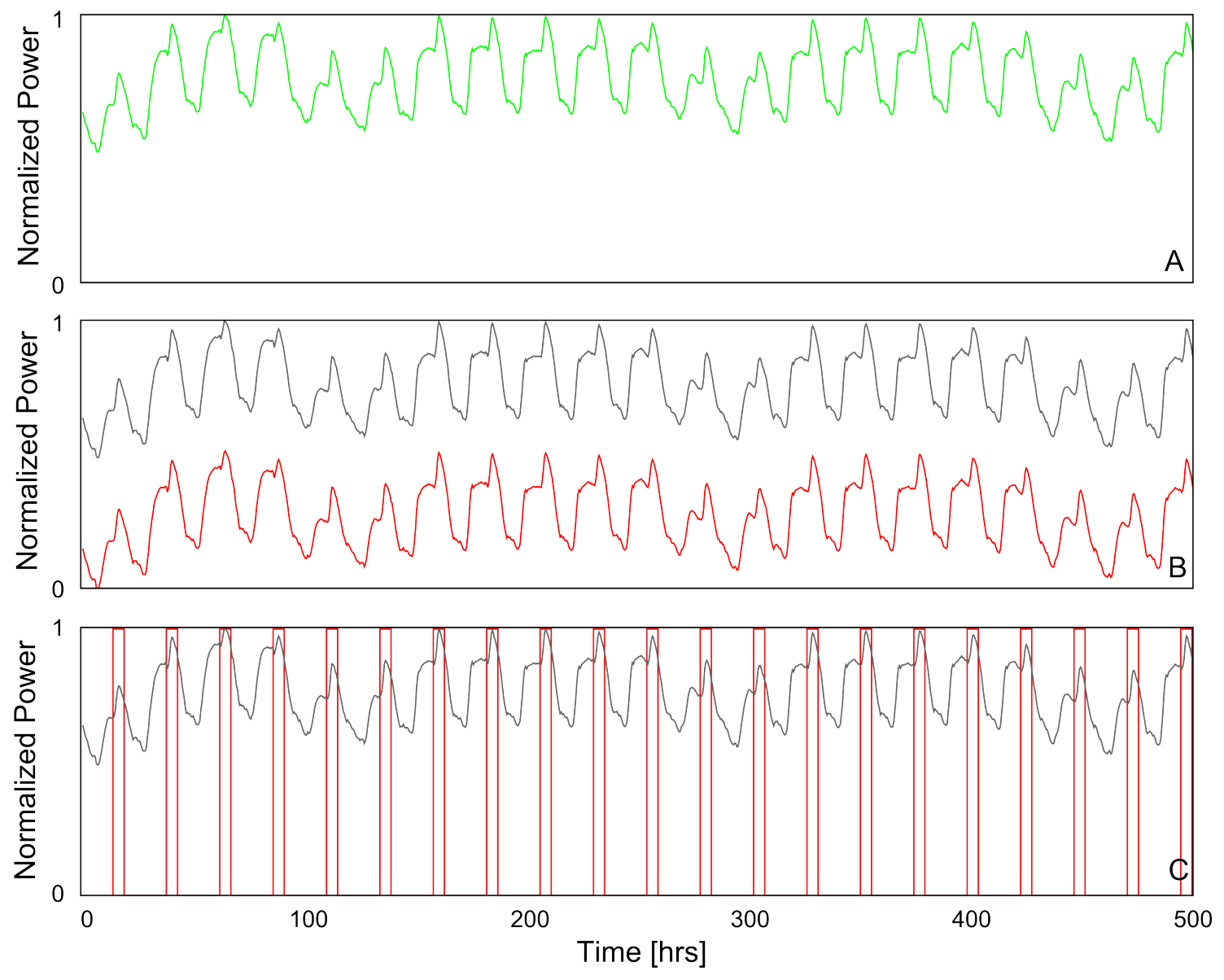

Figure 6: Examples of possible supply profiles. (A) A typical normalized grid demand profile (same as in Figure 1). (B) The same demand with the baseload subtracted to produce a grid minus baseload (GMB) scenario (red), plotted along with the normalized grid demand (gray) for reference. (C) A 5-hour square wave (SW) supply profile centered around the daily peak power consumption. 

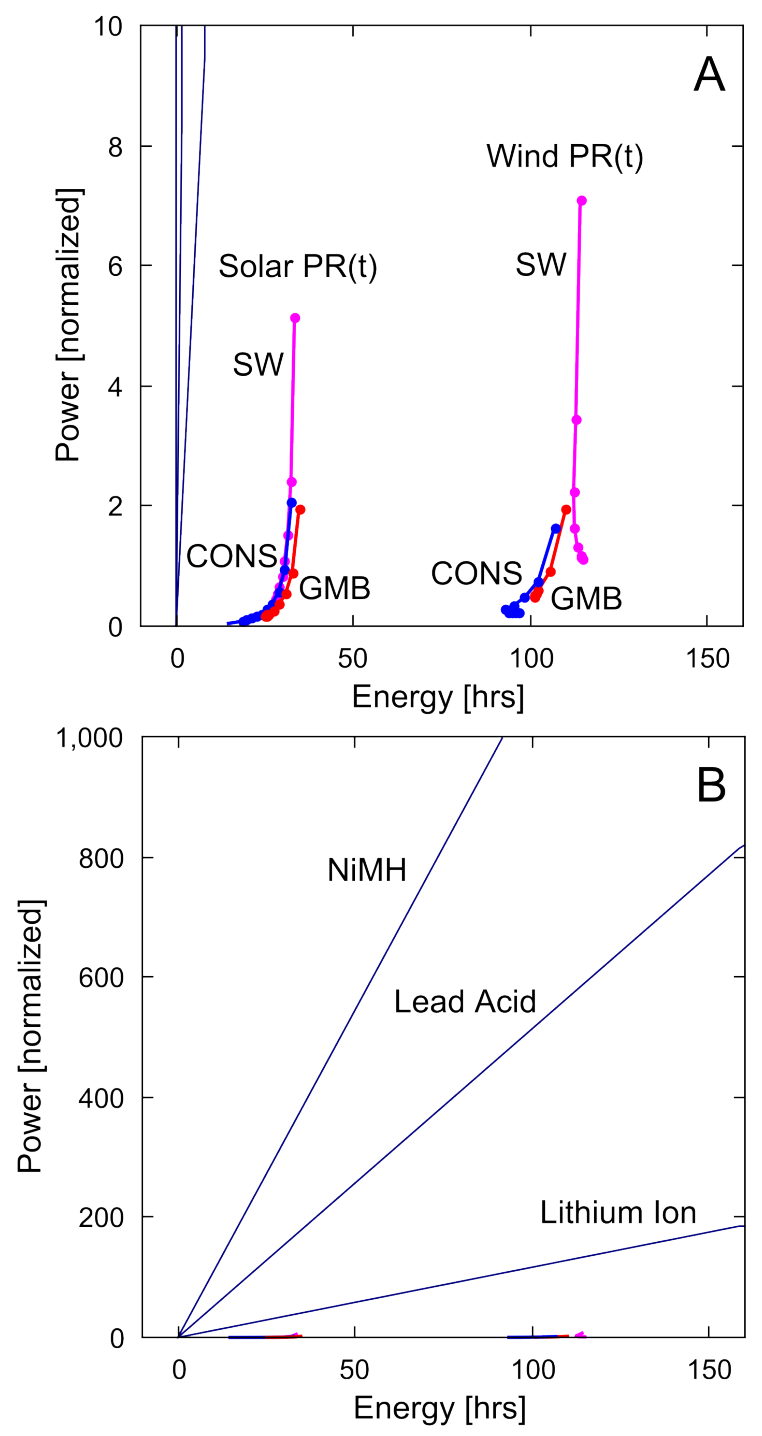

Figure 7: (A) Power and energy requirements, normalized to peak wind or PV production capacity, for several supply scenarios. All the results for the solar PV are grouped to the left below $50 \mathrm{hrs}$. All the results for wind are grouped to the right near $100 \mathrm{hrs}$. In different colors are the different $S U(t)$ scenarios; SW in pink, GMB in red, and CONS in blue. The highest point on each line corresponds to $95 \%$ system efficiency, and the efficiency decreases in $5 \%$ increments with each successive point downwards. (B) The same exact data plotted with a vertical axis scale, showing the vast difference in power scales between common solid-electrode batteries and intermittent renewable electricity storage requirements. The same lines appear nearly vertical in (A) and are unlabeled. 
the same power and energy capacity as supplying the GMB scenario.

In the real world, the supply scenario does not need to be predetermined indefinitely into the future. It may be varied to reflect changes in market conditions. A producer could suddenly have some new transmission become available, and decide to change from the transmission-utilization-maximizing CONS profile to a presumably more profitable supply profile such as SW.

From Fig. 7 we see that the type of production matters much more for the storage energy requirements than does the supply scenario. The power required to reach a given efficiency, however, depends more significantly on the supply scenario than on the production type. We conjecture that this trend is likely to remain valid for all other reasonably desirable supply scenarios.

With this observation we hypothesize some generalizations regarding storage for wind and solar electricity. First, with regard to energy requirements, mapping $1 \mathrm{MW}$ of solar power with the production characteristics of Figure 1B onto any desirable supply scenario requires between about 25 and 50 MWhrs of energy storage capacity (about 1-2 days worth of peak power production capacity). Mapping $1 \mathrm{MW}$ of wind power with the production characteristics of Figure 1A onto any desirable supply scenario requires between about 95 and 115 MWhr of energy storage capacity (about 4-5 days of nameplate power production capacity).

The required storage power is sensitive to the specified system efficiency. If we focus on $\eta_{\text {avg }}=85 \%$ as a reasonably practical goal for the system efficiency, we observe that reaching this goal for $1 \mathrm{MW}$ of either wind or solar PV peak power production requires within a factor of two of $1 \mathrm{MW}$ of storage discharge power 
capacity, depending on the specific supply scenario.

A few representative calculations performed with other wind or solar PV production profiles indicate that these generalizations remain applicable as long as the production capacity factors remain unchanged at $14 \%$ for PV and $32.5 \%$ for wind. An interesting direction for future research is to investigate the sensitivity of these generalizations to variations in capacity factor.

Note that not all system efficiencies are attainable. For example, in Figure $5 \mathrm{~A}$, the two curves terminate at low power instead of extending all of the way down to the origin. As the maximum storage discharge power is decreased, the efficiency decreases. But the maximum discharge power can be decreased only to the point that it reaches the maximum discharge power required in the specific supply scenario, i.e. $\operatorname{MAX}(S T(t)) \leq \Pi A$. The $P_{\text {loss }}(t)$ versus $\Pi A$ curve terminates when this inequality is violated. Certainly it is possible to run at lower efficiency by running at a current density past the value for maximum power, but doing so would achieve lower efficiency and lower power, making such operation useless.

\subsection{Solid Electrode Batteries for Wind or Solar?}

It is interesting to compare the power-energy relationship for typical solid-electrode secondary batteries to the requirements for storing wind and PV electricity. These batteries typically have the characteristic that the power capacity and energy capacity scale together. Nickel metal-hydride batteries, for example, have a specific

power of about $600 \frac{\mathrm{W}}{\mathrm{kg}}$, and a specific energy of about $55 \frac{\mathrm{Whr}}{\mathrm{kg}}$, resulting in an energy to power ratio of roughly $0.1 \mathrm{hr}$., or 6 minutes: this corresponds to the discharge 
time if discharge power could remain at its peak value throughout the process. Similarly, the energy to power ratio of lead acid batteries is roughly $0.2 \mathrm{hr}$., or 12 minutes, and that of lithium ion batteries is $0.6 \mathrm{hr}$, or 35 minutes.

This ratio defines a sloped line through the origin in a power versus energy plot. Moving along the line to larger powers and energies can be thought of as installing larger batteries, or more of them. Plotted along with the storage requirements in Figure 7A are three sloped lines representing the energy to power ratio of NiMH, lead acid, and lithium ion batteries. They are difficult to see, because on this scale the lines are nearly vertical! Figure 7B shows the exact same plot except the vertical axis has been rescaled by a factor of 100 . Now the batteries' energy to power ratios can be observed and compared readily, but the storage requirements have merged with the horizontal axis. We conclude that solid-electrode batteries store about two orders of magnitude too little energy when their power is matched to the storage requirements for wind and solar PV. This does not mean they cannot do the job; on the contrary, if one bought enough battery energy to serve one of these storage scenarios, the batteries would be so vastly overpowered-by two orders of magnitude- that the efficiency would be essentially $100 \%$. The cost of such an overpowered storage system has kept it from broad implementation so far.

Flow batteries and regenerative fuel cells have a significant advantage in this regard. The power and energy capacities of these systems are separate engineering choices. The power capacity is set by the cell hardware, which is typically the expensive part. The energy capacity is set by the amount of reactant and product 
and the size of their storage tanks one buys. Because of this decoupling, one may independently size the power and energy subsystems to be appropriate for the desired scenario. For example, if the intention were to map 1 MW solar production with $14 \%$ capacity factor onto a constant output at $85 \%$ efficiency, one would need to buy roughly a $0.5 \mathrm{MW}$ regenerative fuel cell; to provide the same service, one would have to buy 175 MW of lead acid batteries.

\section{Summary and Conclusions}

With sufficient electricity storage capacity, any power production profile may be mapped onto any desired supply profile. We have presented a detailed framework describing how to calculate the required storage power as a function of time, for any given power production profile, chosen supply profile, and targeted system efficiency, accounting fully for the loss characteristics of the storage system. For constant-activity electrochemical cells, such as the regenerative hydrogen-chlorine fuel cell, a linear approximation of the cell potential versus current density allows the entire efficiency behavior of the system to be described by a single scalar figure of merit-the maximum discharge power $\Pi A$, given by Equation 14. The parameterization in terms of the maximum discharge power of other, non-electrochemical, constant-activity energy storage systems exhibiting nearly linear response may be an equally valuable simplification of their analyses.

We considered three disparate supply scenarios; constant, grid-minus-baseload, and square wave, and two different production scenarios; wind with a capacity 
factor $32.5 \%$, and PV with a capacity factor of $14 \%$. For each of these six combinations of scenarios, we found the storage energy and power capacity requirements for a range of system efficiencies. We found diminishing efficiency returns on increasing the maximum discharge power, as would be expected as one approaches $100 \%$ efficiency. The storage discharge power capacity requirement for a given system efficiency is not very sensitive to variations in the type of power production (wind vs. solar PV), as shown in Figure 7. The required power capacity increases rapidly with increasing efficiency at the high-efficiency end, as shown in Figure 5, illustrating the diminishing returns associated with over-sizing storage power capacity.

The storage energy capacity requirement for a wind production scenario is much more demanding than for a PV production scenario with the same peak production power. The energy requirement is insensitive to the chosen supply scenario or the system efficiency, for the range of system efficiencies studied (70\%95\%). Most of the increased energy demand for wind storage arises from the higher capacity factor of wind compared to that of PV, but some of the increase arises from the characteristic long periods during which the wind is still.

Solid-electrode batteries are shown to have two orders of magnitude too little energy to power ratio to be well suited to storage of intermittent renewables. For both wind and PV, for all the different supply scenarios studied, installing $1 \mathrm{MW}$ of storage discharge capacity for $1 \mathrm{MW}$ of peak production yields system efficiencies of $70 \%-90 \%$. 


\section{Acknowledgments}

This research was supported by National Science Foundation grant NSF-IIP-0848366 through Sustainable Innovations, LLC.

\section{References}

[1] I. P. Gyuk and S. Eckroad. EPRI-DOE Handbook Supplement of Energy Storage for Grid Connected Wind Generation Applications. Technical Report 1008703, EPRI and DOE, 2004.

[2] Koninklijk Nederlands Meteorologisch Instituut. KNMI HYDRA project website.

[3] J. W. Verkaik. Evaluation of Two Gustiness Models for Exposure Correction Calculations. Journal of Applied Meteorology, 39:1613-1626, 2000.

[4] General Electric webpage containing turbine specifications.

[5] NREL PVWatts solar data for Boston, Massachusetts, 2009.

[6] The National Grid webpage where one may download grid consumption data.

[7] B. Parsons, M. Milligan, B. Zavadil, D. Brooks, B. Kirby, K. Dragoon, and J. Caldwell. Grid Impacts of Wind Power: A Summary of Recent Studies in the United States. Technical report, NREL, 2003. 
[8] Jay Apt. The spectrum of power from wind turbines. Journal of Power Sources, 169:369-374, 2007.

[9] Jason Rugolo. Electricity Storage and the Hydrogen-Chlorine Fuel Cell. PhD thesis, Harvard University, 2010.

[10] Bottling Electricity: Storage as a Strategic Tool for Managing Variability and Capacity Concerns in the Modern Grid. Technical report, The Electricity Advisory Committee, DOE, December 2008. 